\title{
Establishing a Dose-Response Relationship Between Opioid Use and Hypogonadism: A Retrospective Case-Control Study
}

\author{
Yashar Eshraghi, MD, ${ }^{1,2}$ Natalie Hanks, MD, MS, ${ }^{2}$ Scott Rooney, MD, ${ }^{1}$ Faeqeh Mir Yousefi Ata, MD, ${ }^{3}$ \\ Cruz Velasco, PhD, ${ }^{4}$ Maged Guirguis, MD, ${ }^{1,2}$ Gabriel Uwaifo, MD $^{2,5}$ \\ ${ }^{1}$ Department of Anesthesiology, Interventional Pain Management, Ochsner Clinic Foundation, New Orleans, LA ${ }^{2}$ The University of \\ Queensland Faculty of Medicine, Ochsner Clinical School, New Orleans, LA ${ }^{3}$ Department of Academics, Faculty of Medicine, Eskisehir \\ Osmangazi University, Eskisehir, Turkey ${ }^{4}$ Center for Applied Health Services Research, Ochsner Clinic Foundation, New Orleans, LA \\ ${ }^{5}$ Department of Endocrinology, Diabetes, Metabolism and Weight Management, Ochsner Clinic Foundation, New Orleans, LA
}

Background: Opioid-induced androgen deficiency (OPIAD) related to chronic, long-acting opioid use can be a significant detriment to patient quality of life. The aim of this study was to investigate the association between chronic opioid use and hypogonadism.

Methods: A single-center, retrospective, matched case-control analysis of 357 males (94 cases, 263 controls, aiming for 1:4 matching) was performed. Study subjects were ages 18 to 80 years and had a diagnosis of chronic opioid use. Patients with a hypogonadism diagnosis were matched to patients without a hypogonadism diagnosis by age, ethnicity, and body mass index. The maximum morphine equivalent daily dose (MEDD) was compared in each group.

Results: A significant linear association between MEDD and the odds of developing hypogonadism $(P<0.001)$ in males with chronic use of opioids was observed, with an odds ratio of 1.44 (95\% Cl 1.16-1.78) by 100-unit difference in maximum MEDD.

Conclusion: Results showed a significant, positive linear association between chronic opioid dose and the odds of developing hypogonadism in males. This higher index of suspicion of OPIAD could lead to earlier recognition of symptoms and increase the positive predictive value of diagnostic laboratory tests.

Keywords: Analgesics-opioid, hypogonadism, opioid-related disorders

Address correspondence to Yashar Eshraghi, MD, Department of Anesthesiology, Interventional Pain Management, Ochsner Clinic Foundation, 2820 Napoleon Ave., New Orleans, LA 70115. Tel: (866) 624-7637. Email: yashar.eshraghi@ochsner.org

\section{INTRODUCTION}

One of the less commonly appreciated, detected, and recognized complications of opioid use is hypogonadotropic hypogonadism. Opioid-induced androgen deficiency (OPIAD) was first documented in 1973, when males addicted to heroin or methadone were noted to have lower levels of testosterone. ${ }^{1}$ More recent studies have continued to demonstrate a high rate of both hypotestosteronemia and clinically significant hypogonadism in males taking opioids for chronic pain or maintenance therapy for addiction. ${ }^{2-5}$ Although many opioid adverse effects are well known and readily identified in clinic, the effect opioids have on the endocrine system are commonly overlooked and underappreciated, even though their prevalence is likely fairly high with potential quality of life and health-related consequences. ${ }^{6-9}$

The negative side effects of chronic opioid use on the endocrine system are significant in the adult population but not adequately characterized in the medical literature. Androgen deficiency, which is one of the more dominant endocrine consequences of chronic opioid use, has serious morbidity potential, including fatigue, muscle atrophy (sarcopenia), decreased libido, erectile dysfunction, depressed mood with anhedonia, reduced quality of life, anemia, subfertility or infertility, gynecomastia, and decreased bone density, which can lead to osteoporosis and bone fractures. ${ }^{10,11}$ These complications can cause substantive distress for patients and are major expenses for health care systems. ${ }^{12,13}$ As narcotic abuse continues to rise, elucidating the consequences of continuous opioid use becomes crucial. Studies to date lack the statistical power to adequately investigate a dose-response relationship between opioids and hypogonadism. Examining this relationship is necessary to help identify which patients on opioids need to be screened for these complications and to guide adequate and safe treatment of such patients. The dose-response relationship 


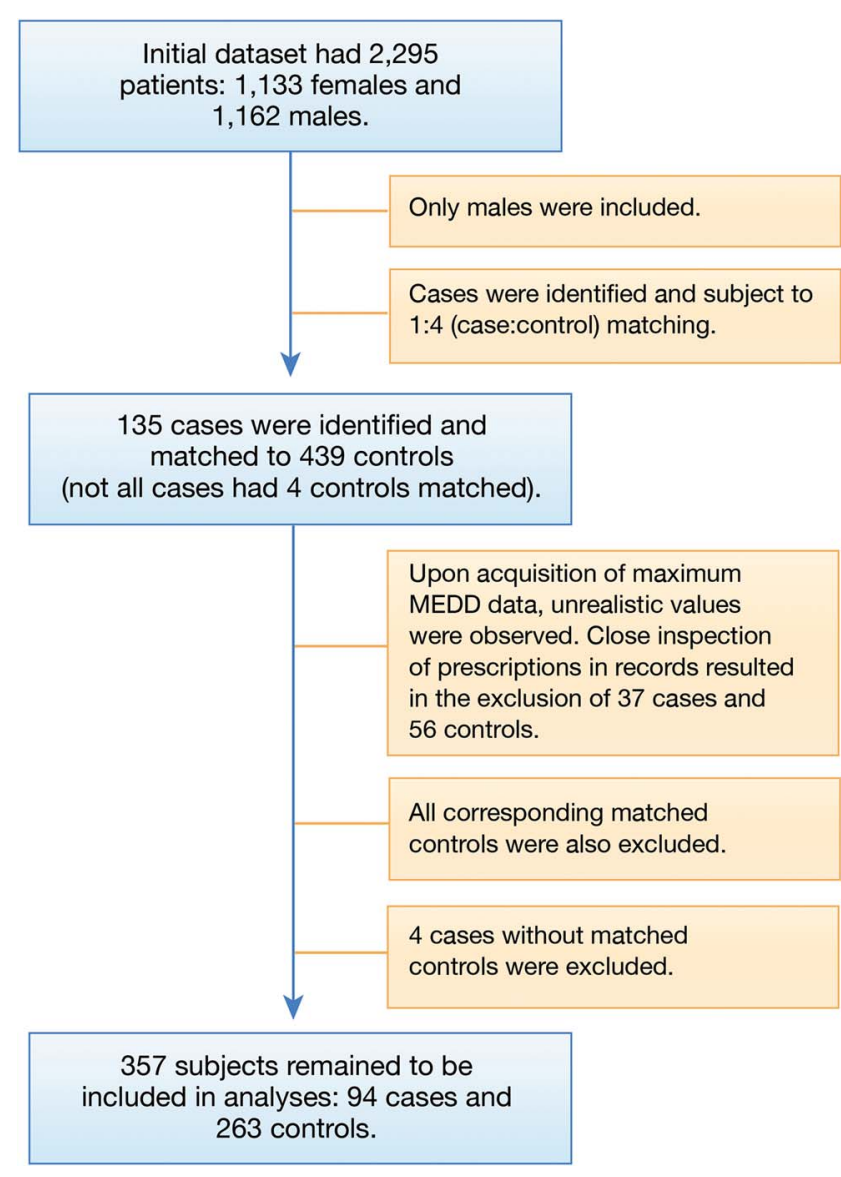

Figure 1. Data flow diagram demonstrates subject selection for the study. MEDD, morphine equivalent daily dose.

between opioids and hypogonadism could also reshape clinical guidelines for the diagnosis, management, and/or treatment of OPIAD.

With this retrospective medical record review, we aimed to characterize the prevalence of male hypogonadism among chronic opioid users and the dose-effect relationship between opioids and hypogonadism.

\section{METHODS \\ Study Design}

This retrospective case-control study using health data from the Ochsner Health Epic electronic health record (EHR) database was approved by the Institutional Review Board of Ochsner Health with waiver of consent (\#STUDY00000714). Patients' medical records were reviewed to collect data on demographics, medications, and inpatient and outpatient diagnoses.

\section{Study Population}

We identified 1,162 males aged 18 to 80 years who had $\geq 90$ days of opioid use from January 2012 to May 2019 (Figure 1). The cutpoint of $\geq 90$ days was selected because it is a well-established clinical threshold of prolonged opioid use. We excluded males who had a diagnosis of Klinefelter syndrome, other chromosomal abnormalities, cryptorchidism, varicocele, myotonic dystrophy, prior mumps infection, radiation to the testes, testicular torsion, long-term corticosteroid use, prostate cancer, or other known relevant endocrine disorder. These exclusions narrowed the cohort to 574 subjects. Of the 574 subjects, 135 carried a chart diagnosis of hypogonadism or low testosterone. Patients were excluded from the study if they were diagnosed with hypogonadism or low testosterone before their chronic opioid use.

For the control group, we identified 439 subjects who never had a diagnosis of hypogonadism or low testosterone and followed the aforementioned exclusion criteria. Cases were matched (aiming for 1:4 matching) to controls by age, ethnicity, and body mass index (BMI). We used the greedy method, which matches one or more controls for each case; controls may be matched to cases by one or more covariates. Once a match is made using this method, it cannot be broken. The greedy method was implemented by using the SAS macro gmatch ${ }^{14}$ (matching weights $=3,2,1$ and largest difference compatible with a valid match $=0,1,3$ for race, age, and BMI, respectively, and with weighted sum distance). Four cases did not have matching controls, so they were excluded from the study.

\section{Measurements}

The Ochsner Epic EHR automatically calculates the morphine equivalent daily dose (MEDD) for each opioid prescription. After examination of the MEDD data for the cohort, we found several MEDDs to be $>10,000$. Each $>10,000$ value was associated with a prescription for either methadone or buprenorphine. Because methadone increases in potency with opioid-tolerant patients and buprenorphine acts as a partial opioid agonist, calculating the MEDD varies. Because of these additional complexities, patients taking methadone or buprenorphine were excluded from the study, resulting in the exclusion of 37 cases and 56 controls. After matching, a total cohort of 357 subjects was used in the study: 94 cases and 263 controls.

\section{Statistical Analysis}

The observed prevalence of hypogonadism by groups of matching variables (before matching) and for ranges of MEDD postmatch were calculated. The association between hypogonadism and MEDD was evaluated by conditional logistic regression, with linear terms of continuous MEDD. To account for confounding variables, we matched cases to controls by age, ethnicity, and BMI. A nonlinear association of MEDD with hypogonadism was illustrated with restricted cubic splines. Analyses were performed using SAS/STAT statistical software, version 14.2 (SAS Institute, Inc). This manuscript adheres to the application of the Strengthening the Reporting of Observational Studies in Epidemiology (STROBE) guidelines.

\section{RESULTS}

Table 1 displays the prevalence of hypogonadism among the total 1,162 male subjects with chronic opioid use by age, race, and BMl groups. Among subjects in the age groups of 18 to 39 years, 40 to 65 years, and $>65$ years, $7.2 \%, 13.7 \%$, and $13.1 \%$, respectively, were diagnosed with hypogonadism. Among subjects diagnosed with hypogonadism, 3.8\% were underweight (BMl $<18.5 \mathrm{~kg} / \mathrm{m}^{2}$ ), $11.4 \%$ were normal weight (BMI 18.5 to $24.9 \mathrm{~kg} / \mathrm{m}^{2}$ ), $10.9 \%$ were overweight (BMI of 25 to $29.9 \mathrm{~kg} / \mathrm{m}^{2}$ ), and $15.1 \%$ were obese $\left(\mathrm{BMI}>30 \mathrm{~kg} / \mathrm{m}^{2}\right)$. 
Table 1. Prevalence of Hypogonadism in the Study Cohorts by Age, Race, and Body Mass Index

\begin{tabular}{|c|c|c|c|}
\hline Variable & $\begin{array}{l}\text { All Patients, } \\
n=1,162^{a}\end{array}$ & $\begin{array}{l}\text { Patients Without } \\
\text { Hypogonadism, } \\
n=1,016^{b}\end{array}$ & $\begin{array}{c}\text { Patients With } \\
\text { Hypogonadism, } \\
n=146^{b}\end{array}$ \\
\hline \multicolumn{4}{|l|}{ Age, years } \\
\hline $18-39$ & $167(14.4)$ & $155(92.8)$ & $12(7.2)$ \\
\hline $40-65$ & $621(53.4)$ & $536(86.3)$ & 85 (13.7) \\
\hline$>65$ & $374(32.2)$ & $325(86.9)$ & $49(13.1)$ \\
\hline \multicolumn{4}{|l|}{ Race } \\
\hline White & $828(71.3)$ & $723(87.3)$ & $105(12.7)$ \\
\hline Black & $269(23.1)$ & $231(85.9)$ & $38(14.1)$ \\
\hline Other & $65(5.6)$ & $62(95.4)$ & $3(4.6)$ \\
\hline \multicolumn{4}{|l|}{ Body mass index, $\mathrm{kg} / \mathrm{m}^{2}$} \\
\hline Underweight, $<18.5$ & $26(2.2)$ & $25(96.2)$ & $1(3.8)$ \\
\hline Normal, 18.5-24.9 & $263(22.6)$ & $233(88.6)$ & $30(11.4)$ \\
\hline Overweight, 25-29.9 & $396(34.1)$ & $353(89.1)$ & $43(10.9)$ \\
\hline Obese, $\geq 30$ & $477(41.0)$ & $405(84.9)$ & $72(15.1)$ \\
\hline Obese Class 1, 30-<35 & $271(23.3)$ & $234(86.3)$ & 37 (13.7) \\
\hline Obese Class 2, 35-<40 & $122(10.5)$ & $102(83.6)$ & $20(16.4)$ \\
\hline Obese Class $3, \geq 40$ & $84(7.2)$ & $69(82.1)$ & 15 (17.9) \\
\hline
\end{tabular}

apercentages are calculated by column.

${ }^{b}$ Percentages are calculated across the row.

Note: Data are presented as $\mathrm{n}(\%)$.

From the conditional logistic regression with continuous MEDD as a predictor, we observed a linear association of developing hypogonadism $(P<0.001)$ with an odds ratio of $1.44(95 \% \mathrm{Cl} 1.16-1.78)$ for each 100 -unit difference in MEDD. Thus, we observed 44\% (95\% Cl 16\%$78 \%$ ) increased odds of developing hypogonadism between patients having a 100-unit difference in MEDD.

Table 2 shows the observed prevalence of hypogonadism for intervals of MEDD. The plateau from 400 to 800 MEDD is based on a limited sample size $(n=14)$. From a logistic regression model with restricted cubic splines on MEDD, we found a nonlinear association with the odds of hypogonadism status $(P=0.015)$. Figure 2 depicts the nonlin-

Table 2. Prevalence of Hypogonadism for Each Morphine Equivalent Daily Dose (MEDD) Range

\begin{tabular}{lccc}
\hline MEDD Range & $\begin{array}{c}\text { Total Patients, } \\
\mathbf{n = 3 5 6}\end{array}$ & $\begin{array}{c}\text { With Hypogo- } \\
\text { nadism, } \\
\mathbf{n = 9 3 ^ { \mathbf { a } }}\end{array}$ & $\begin{array}{c}\text { Control Group } \\
\text { Without } \\
\text { Hypogo- } \\
\text { nadism, } \\
\mathbf{n = 2 6 3}\end{array}$ \\
\hline $0-99$ & 135 & $21(15.6)$ & $114(84.4)$ \\
$100-199$ & 140 & $38(27.1)$ & $102(72.9)$ \\
$200-299$ & 53 & $22(41.5)$ & $31(58.5)$ \\
$300-399$ & 14 & $5(35.7)$ & $9(64.3)$ \\
$400-800$ & 14 & $7(50.0)$ & $7(50.0)$ \\
\hline
\end{tabular}

a One patient had a MEDD $>800$ and is not included in the analysis. Note: Percentages are calculated by row. Data are presented as $n$ (\%). ear association as an increase and plateau of the estimated risk of hypogonadism for intervals of MEDD. Figure 3 demonstrates the same estimated risk of hypogonadism as a smoothed function of continuous MEDD. Both Figures 2 and 3 show an increased estimated risk of hypogonadism as the MEDD increases.

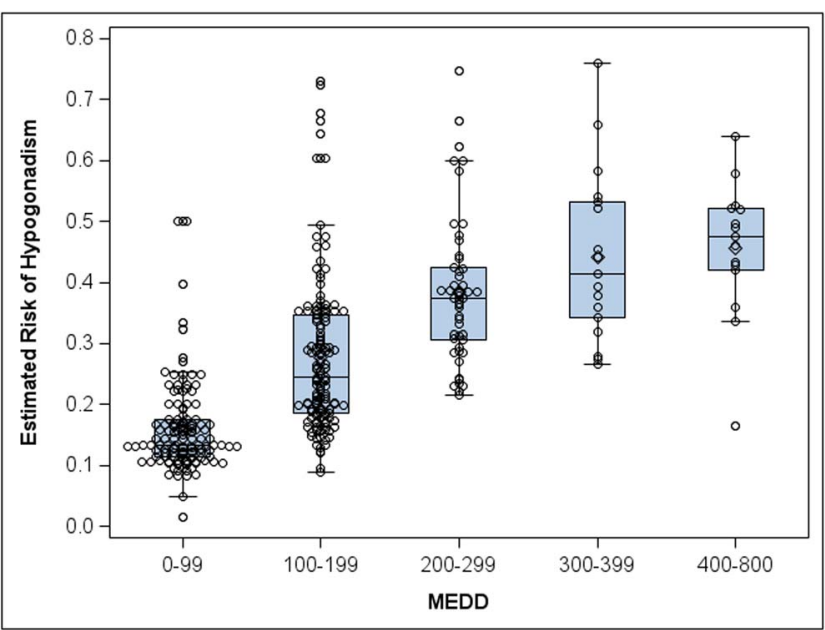

Figure 2. Estimated risk of hypogonadism for patients at various ranges of morphine equivalent daily dose (MEDD). The circles are an estimated risk of hypogonadism for each subject from a conditional logistic regression model on MEDD. The figure displays a rise followed by a plateau in average risk of hypogonadism with increasing MEDD. 


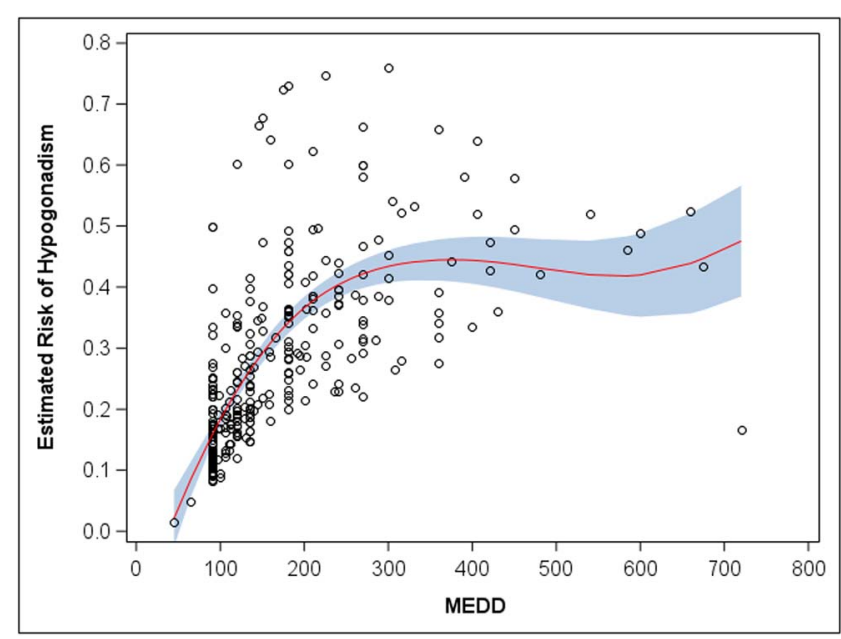

Figure 3. Estimated risk of hypogonadism as a function of continuous morphine equivalent daily dose (MEDD). The circles are an estimated risk of hypogonadism for each subject from a conditional logistic regression model on MEDD. The red line is the average estimated risk of hypogonadism as a function of MEDD. The blue band represents the confidence intervals of estimated risk. Wider confidence intervals reflect smaller sample sizes and thus less certainty about the average estimated risk of hypogonadism. (A color version of this figure is available at https://doi.org/10.31486/toj.20.0103.)

\section{DISCUSSION}

Our primary results show that chronic opioid users were more likely to have hypogonadism with higher daily doses of prescribed opioids. This positive trend was highly statistically significant. Our findings are consistent with prior studies demonstrating an increased prevalence of androgen deficiency among chronic opioid users, but to our knowledge, this study is the first to demonstrate a significant doseeffect relationship between opioids and the development of hypogonadism. ${ }^{10}$ As is apparent from our findings and previous studies, at least a fraction of this observed association between hypogonadism and opioid use is causal and therefore amenable to intervention.

The side effects of hypogonadism related to chronic opioid use can be a significant burden to patient quality of life and the health care system. However, the negative sequelae of chronic opioid use on the endocrine system are often poorly investigated, frequently unrecognized, and commonly left untreated..$^{15}$ As of 2021, no guidelines are available for the recognition and diagnosis of OPIAD. Studies have demonstrated an increased prevalence of OPIAD in patients receiving opioid therapy for longer than a few weeks, as well as an increased prevalence in patients receiving a median dose >100 MEDD. ${ }^{16,17}$ Evaluation of any patient manifesting symptoms of hypogonadism for signs of OPIAD, such as reduced libido, erectile dysfunction, depression, fatigue, or night sweats, is appropriate. Laboratory testing to assess for the presence of opioid-induced hypogonadism, including total testosterone, free testosterone, sex hormone binding globulin, luteinizing hormone, follicle-stimulating hormone, dehydroepiandrosterone sulfate, and estradiol, may be warranted if clinical suspicion is high enough. Treatment options include lifestyle changes in diet and exercise, tapering of opi- oids, opioid rotation, or hormonal replacement therapies if symptoms are significant enough. $3,18,19$

Although OPIAD is studied primarily in the male population, future studies should expand the patient population examined to include females. Opioid-induced hypogonadism affects the female population in similar ways as males. Females have comparable symptoms, as well as hot flashes and irregular menstruation. Examining if a doseresponse relationship between chronic long-acting opioid use and hypogonadism exists in females could be important. Because this study demonstrates a positive correlation between the daily dose of prescribed opioids and the prevalence of hypogonadism, clinicians should have an increased index of suspicion for the development of hypogonadism in patients using opioids chronically. This higher index of suspicion could lead to earlier recognition of symptoms, increase the positive predictive value of diagnostic laboratory tests, and potentially assist in the formation of clinical guidelines for the diagnosis and management of OPIAD.

Important limitations of this study include its retrospective nature and the inability to document causality between opioid exposure and development of OPIAD. The retrospective nature of our study also limits our capacity to rigorously distinguish between low testosterone as a biochemical finding vs clinical hypogonadism as a clinical syndrome. MEDD data were taken from the EHR and may not represent the dosages patients were taking. The degree to which OPIAD can impact both short-term and long-term fertility, especially in younger patients using opioids, is another important clinical question that our study was not designed to answer. Finally, although our study includes one of the largest reported cohorts in this area of investigation, the sample size is still limited relative to the patient population exposed to opioids. Our study was not able to characterize the role and effects of methadone and buprenorphine use, as patients taking these drugs were excluded from our cohort, but these opioid receptor partial agonists are widely used in the chronic opioid abuse remediation population. These limitations highlight the urgent need for well-designed, possibly multicenter, prospective clinical studies to investigate these and other questions regarding OPIAD in both males and females on chronic opioid therapy.

\section{CONCLUSION}

This single-center study demonstrates the linear doseresponse relationship between chronic opioid use and hypogonadism. Recognition of this relationship leads to earlier recognition of symptoms, increases the positive predictive value of diagnostic laboratory tests, and potentially assists in developing formal clinical guidelines for the diagnosis and management of OPIAD in future studies.

\section{ACKNOWLEDGMENTS}

The authors have no financial or proprietary interest in the subject matter of this article.

\section{REFERENCES}

1. Azizi F, Vagenakis AG, Longcope C, Ingbar SH, Braverman LE. Decreased serum testosterone concentration in male heroin and methadone addicts. Steroids. 1973;22(4):467-472. doi: $10.1016 / 0039-128 \times(73) 90002-0$ 
2. Hedegaard H, Bastian BA, Trinidad JP, Spencer M, Warner M. Drugs most frequently involved in drug overdose deaths: United States, 2011-2016. Centers for Disease Control and Prevention. December 12, 2018. Accessed March 10, 2021. www.cdc.gov/nchs/data/nvsr/nvsr67/nvsr67_09-508.pdf

3. O'Rourke TK Jr., Wosnitzer MS. Opioid-induced androgen deficiency (OPIAD): diagnosis, management, and literature review. Curr Urol Rep. 2016;17(10):76. doi: 10.1007/s11934-016-0634-y

4. Rajagopal A, Vassilopoulou-Sellin R, Palmer JL, Kadur G, Bruera E. Hypogonadism and sexual dysfunction in male cancer survivors receiving chronic opioid therapy. J Pain Symptom Manage. 2003;26(5):1055-1061. doi: 10.1016/s0885-3924(03)00331-2

5. Rubinstein AL, Carpenter DM, Minkoff JR. Hypogonadism in men with chronic pain linked to the use of long-acting rather than short-acting opioids. Clin J Pain. 2013;29(10):840-845. doi: 10.1097/AJP.0b013e31827c7b5d

6. Schug SA, Zech D, Grond S. Adverse effects of systemic opioid analgesics. Drug Saf. 1992;7(3):200-213. doi: 10.2165/00002018-199207030-00005

7. Haddox JD, Joransen D, Angarola RT, et al. The use of opioids for the treatment of chronic pain. A consensus statement from the American Academy of Pain Medicine and the American Pain Society. Clin J Pain. 1997;13(1):6-8.

8. Kolodny A, Courtwright DT, Hwang CS, et al. The prescription opioid and heroin crisis: a public health approach to an epidemic of addiction. Annu Rev Public Health. 2015;36:559-574. doi: 10.1146/annurev-publhealth-031914-122957

9. Elliott JA, Horton E, Fibuch EE. The endocrine effects of long-term oral opioid therapy: a case report and review of the literature. J Opioid Manag. 2011;7(2):145-154. doi: 10.5055/jom.2011.0057

10. Daniell HW. Hypogonadism in men consuming sustained-action oral opioids. J Pain. 2002;3(5):377-384. doi: 10.1054/jpai.2002.126790
11. Daniell HW. Opioid osteoporosis. Arch Intern Med. 2004;164(3):338; author reply, 338. doi: $10.1001 /$ archinte.164.3.338-a

12. Drug Abuse Warning Network, 2011: national estimates of drug-related emergency department visits. U.S. Department of Health and Human Services Substance Abuse and Mental Health Services Administration Center for Behavioral Health Statistics and Quality. Accessed March 10, 2021. www.samhsa. gov/data/sites/default/files/DAWN2k11ED/DAWN2k11ED/ DAWN2k11ED.pdf

13. Patrick SW, Schumacher RE, Benneyworth BD, Krans EE, McAllister JM, Davis MM. Neonatal abstinence syndrome and associated health care expenditures: United States, 2000-2009. JAMA. 2012;307(18):1934-1940. doi: 10.1001/jama.2012.3951

14. gmatch. Kosanke J, Bergstralh E; 2004. Accessed June 2016. bioinformaticstools.mayo.edu/downloads/sas/gmatch.sas

15. Katz N, Mazer NA. The impact of opioids on the endocrine system. Clin J Pain. 2009;25(2):170-175. doi: 10.1097/AJP.0b013e3181850df6

16. Aloisi AM, Pari G, Ceccarelli I, et al. Gender-related effects of chronic non-malignant pain and opioid therapy on plasma levels of macrophage migration inhibitory factor (MIF). Pain. 2005;115(1-2):142-151. doi: 10.1016/j.pain.2005.02.019

17. Colameco S, Coren JS. Opioid-induced endocrinopathy. J Am Osteopath Assoc. 2009;109(1):20-25.

18. Heufelder AE, Saad F, Bunck MC, Gooren L. Fifty-two-week treatment with diet and exercise plus transdermal testosterone reverses the metabolic syndrome and improves glycemic control in men with newly diagnosed type 2 diabetes and subnormal plasma testosterone. J Androl. 2009;30(6):726-733. doi: 10.2164/jandrol.108.007005

19. Brennan MJ. The effect of opioid therapy on endocrine function. Am J Med. 2013;126(3 Suppl 1):S12-S18. doi: 10.1016/j.amjmed.2012.12.001

This article meets the Accreditation Council for Graduate Medical Education and the American Board of Medical Specialties Maintenance of Certification competencies for Patient Care, Medical Knowledge, and Practice-Based Learning and Improvement.

C2021 by the author(s); licensee Ochsner Journal, Ochsner Clinic Foundation, New Orleans, LA. This article is an open access article distributed under the terms and conditions of the Creative Commons Attribution (CC BY) license (creativecommons.org/licenses/by/4.0/legalcode) that permits unrestricted use, distribution, and reproduction in any medium, provided the original author(s) and source are credited. 